\section{Estructura familiar y estado de nutrición en adolescentes de Sonora, México}

\author{
CRUZ MÓNICA LÓPEZ-MORALES ${ }^{1}$, ADEMIR LÓPEZ-VALENZUELA ${ }^{2}$, \\ RICARDO GONZÁLEZ-HEREDIA ${ }^{2}$, OLGA ROSA BRITO-ZURITA ${ }^{3}$, \\ EUSEBIO ROSALES-PARTIDA ${ }^{4}$, GABRIEL HERNANDO PALOMARES-URIBE ${ }^{2}$
}

\section{Family structure of obese/overweight and normal weight adolescents in México}

Background: Feeding modes and appetence toward certain foods are usually conditioned by the family. Obesity during adolescence usually persists during adulthood. Aim: To determine differences in family structure of adolescents according to their nutritional status. Material and Methods: A cross-sectional study was conducted in 60 overweight-obese and 60 normal weight adolescents. Family type was determined based on their conformation (kinship and cohabitation), development (if the mother had a remunerated job), demography (geographical area), integration (functions of the couple); life cycle stage and functionality. Results: Fifty eight percent of normal weight adolescents had simple nuclear families and $47 \%$ of overweight-obese adolescents had an extended family. Thirty one and $21 \%$ of overweight/obese and normal weight adolescents lived with an overweight/obese individual, respectively. Conclusions: There are differences in the family structure of overweight/obese and normal weight adolescents.

(Rev Med Chile 2016; 144: 181-187)

Key words: Adolescent; Family relation; Obesity.

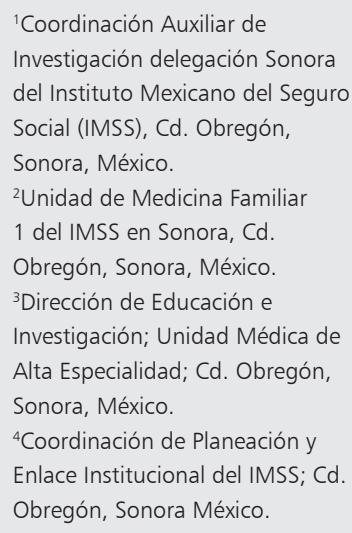

Correspondencia a:

Cruz Mónica López-Morales 5 de Febrero 220 nte. Colonia Centro, Cd. Obregón, Sonora; México.

Teléfono: (644) 1012525 monica.lopezm26@gmail.com
L os modos de alimentarse, preferencias y rechazos hacia determinados alimentos están fuertemente condicionados por el contexto familiar durante la etapa infantil, en la cual se incorporan la mayoría de los hábitos y prácticas alimentarias ${ }^{1}$.

La familia se ubica dentro de un sistema socio-económico-cultural de una sociedad "El término familia abarca una enorme extensión de características, conductas y experiencias"; es el núcleo primario de la sociedad y del contexto social de la salud ${ }^{2,3}$ y el primer contacto del individuo respecto a comportamientos en todos los aspectos, incluyendo la alimentación, ya que influye de gran manera en el comportamiento de comer del individuo y por lo tanto, en el estado de nutrición y salud del mismo ${ }^{4}$.

Para la Organización Mundial de la Salud, la familia es la unidad básica de la organización social y también la más accesible para efectuar intervenciones preventivas y terapéuticas ${ }^{5}$. La familia puede ser clasificada desde una gran diversidad de características, es posible tipificarla de acuerdo con la etapa del ciclo vital, demografía, psicosocial, dinámica familiar, límites, ocupación del jefe de familia y conformación ${ }^{6,7}$.

Actualmente los cambios económicos, culturales y contextuales sociales obligan también a realizar cambios en el interior y en la estructura familiar, donde la capacidad de adquisición se ha convertido en prioridad, favoreciendo el sedentarismo, la ingesta de comida rápida, el cuidado a cargo de los abuelos y autosuficiencia a edad temprana lo cual ha provocado un aumento exagerado de alteraciones en los estados de nutrición como el sobrepeso y obesidad. 
El estado de nutrición es la situación en la que se encuentra una persona en relación con la ingesta y adaptaciones fisiológicas que tienen lugar tras la ingesta de nutrientes, cuyas alteraciones son multifactoriales, teniendo gran peso el contexto, la disponibilidad de alimentos, cultura y costumbres ${ }^{8}$. El índice de masa corporal (IMC) es un objetivo indicador antropométrico del estado nutricional de la población'; es considerado el mejor indicador antropométrico para diagnosticar el sobrepeso y la obesidad en individuos entre los 2 y los 18 años $^{10}$.

Se estima que $80 \%$ de los adolescentes que actualmente son portadores de obesidad, continuarán en la etapa adulta con este padecimiento, acompañada de diversas complicaciones ${ }^{11}$.

En la población de 12 a 19 años de México, el sobrepeso y obesidad se presentan en 35\%, siendo 2,4 puntos porcentuales mayor el porcentaje en adolescentes de sexo masculino (14,5\%) que en las de sexo femenino $(12,1 \%)^{12}$, cifras que son variables según la región; en el estado de Sonora, México, la prevalencia es mayor a la media nacional, reportando $17,2 \%$ los adolescentes varones contra $13,6 \%$ en las mujeres ${ }^{13,14}$. Lo anterior explicado por la dieta del sonorense, la cual constituye un riesgo, porque es alta en grasas, proteína de origen animal, baja en fibra, vitaminas y minerales, con poca fruta y verdura ${ }^{15}$.

En las regiones urbanas, la oferta de alimentos secundarios y periféricos es mayor y muy diversa. Los patrones alimentarios de la población urbana del estado de Sonora serán tan variados como el acceso económico lo permita; la frecuencia de consumo de frutas y vegetales, a excepción de la cebolla y el tomate, no supera $30 \%$ de la población ${ }^{16,17}$.

Actualmente, la mayoría de los estudios realizados sobre obesidad y tipología familiar se han implementado en niños en edad escolar, utilizando gran diversidad de escalas y clasificaciones, en contextos distintos y sin seguimiento, lo cual origina que la mayoría de los resultados sean contrastantes, poco aplicables, sin determinar, al final de cuentas, el impacto real en la problemática de obesidad.

El presente estudio pretende determinar si existen diferencias en la estructura familiar de los adolescentes según su estado de nutrición, la edad que se contempla es de 15 a 18 años, con la finalidad de controlar la influencia de los cambios hormonales que se desarrollan en esta etapa.

\section{Material y Métodos}

Se llevó a cabo un estudio transversal analítico en 120 adolescentes, con edades entre 15 y 18 años; la muestra se calculó con la fórmula para dos proporciones, considerando un intervalo de confianza de $95 \%$ y un poder de $80 \%$, los cuales se obtuvieron mediante un muestreo por casos consecutivos. Se seleccionaron adolescentes que acudieran a la Unidad de Medicina Familiar 1 en el período de marzo-agosto de 2013 y que cumplieran con los criterios de selección; el grupo de casos fueron los pacientes con sobrepeso-obesidad, cuyo índice de masa corporal se ubicara en el percentil 90 de las tablas de la CDC (Centers for Disease Control and Prevention), con ausencia de malformaciones congénitas, endocrinopatías, discapacidad física que lo obligara a permanecer en silla de ruedas, muletas o reposo obligado por más de tres meses o practicara algún deporte de manera profesional; el grupo control fueron pacientes cuyo IMC se ubicara entre los percentiles 15 y 85 según las tablas de la CDC, lo cual se considera peso normal, considerando los mismos criterios de selección y se organizaron en pares por edad y sexo.

Al localizar al posible participante se le explicó en qué consistía el estudio y se citó entre 7 y 10 de la mañana, acompañado de padre o tutor, los cuales firmaron consentimiento informado; a cada adolescente se le explicó en qué consistía el estudio y sólo se procedió con el consentimiento de él.

Se inició con la somatometría colocando al adolescente en báscula previamente calibrada con un mínimo de ropa, sin calzado, después de haber evacuado y vaciado la vejiga y en ayuno, ubicándolo en el centro de la plataforma, parado de frente al medidor, erguido con hombros abajo, los talones juntos y con las puntas separadas, brazos colocados en los costados y holgados, sin ejercer presión, cabeza firme con vista al frente en un punto fijo.

Para la obtención de la talla, se colocó en el centro la plataforma, parado de espalda al estadímetro, en la misma posición se le solicitó contraer los glúteos; el especialista frente a él colocó ambas manos en el borde inferior del maxilar inferior del explorado, ejerciendo una mínima tracción hacia arriba, vigilando que el sujeto no se colocara de puntillas colocando su mano en las rodillas, las piernas rectas, talones juntos y puntas separadas, 
procurando que los pies formen un ángulo de $45^{\circ}$, se deslizó la escuadra del estadímetro de arriba hacia abajo hasta topar con la cabeza del sujeto, presionando suavemente contra la cabeza para comprimir el cabello y en donde marcaba la línea es lo que se consignó en la hoja de recolección de datos.

Posteriormente se calculó el IMC y se ubicaron en las tablas de percentiles de la CDC considerando edad y sexo; al adolescente que cumpliera los criterios de selección para casos se ingresaba, y junto al tutor se les aplicaba un cuestionario estructurado con datos básicos para determinar la tipología familiar en base a su conformación (parentesco y cohabitación), desarrollo (actividad remunerada de la madre), demografía (área geográfica donde reside la familia: rural y urbana), integración (funciones de cónyuges) y etapa del ciclo vital según la Organización Mundial de la Salud: I. Formación matrimonio; II. Extensión (nacimiento del primer hijo, nacimiento del último hijo); III. Extensión completa (nacimiento del último hijo); IV. Contracción (primer hijo abandona el hogar); V. Contracción completa (último hijo abandona el hogar) y VI. Disolución (muerte del primer cónyuge); se aplicó el APGAR (adaptación, participación, gradiente de recurso personal, afecto, y recursos) familiar para conocer su funcionalidad dicho instrumento (se requiere aplicar a por los menos dos miembros de la familia y mayores de 15 años), cuyas siglas refieren las mediciones de adaptabilidad (adaptability), cooperación (partnertship), desarrollo (growth), afectividad (affection) y capacidad resolutiva (resolve) y se interrogó acerca del antecedente de obesidad en el grupo familiar con o sin parentesco. Los datos se vaciaron en una hoja diseñada para la recolección y manejo de esta información $y$, después en el área de medicina preventiva se procedió a buscar el control par en sexo, edad (con una tolerancia de 6 meses) y de IMC (según la tabla de percentiles de la CDC).

Se empleó el programa SPSS V.18 para el análisis de los datos, mediante frecuencias, medidas de tendencia central, se aplicó $\chi^{2}$ y U de Mann Whitney para determinar la existencia de diferencia entre grupos.

El protocolo fue revisado y autorizado por Comité Local de Investigación y Ética en Investigación en Salud 2603, de la Unidad de Medicina Familiar 1 de Ciudad Obregón, Sonora y se realizó bajo los lineamientos nacionales e internaciones para realizar investigación en seres humanos.

\section{Resultados}

Se analizó una muestra total de 120 adolescentes, los cuales pertenecen a una población de 12.572 adolescentes adscritos a la Unidad de Medicina Familiar 1 del IMSS (Instituto Mexicano del Seguro Social), en Ciudad Obregón, Sonora, de los cuales 60 se incluyeron en el grupo de casos (sobrepeso y obesidad) y 60 en el grupo control (normal), construyendo pares en edad y sexo, quedando 30 hombres y 30 mujeres en cada grupo, para completar los 60 por grupo (Tabla 1).

Con base en encuesta aplicada a adolescentes y tutores de ambos grupos, se determinó la estructura (parentesco, integración, desarrollo, demografía), etapa del ciclo vital en la que se ubican y funcionalidad familiar; encontrándose diferencias estadísticamente significativas en la estructura con base en el parentesco (Tabla 2), predominando la familia nuclear simple en el grupo con control (peso normal) y la extensa en el grupo de casos (con sobrepeso y obesidad IMC > percentil 90).

Respecto al antecedente de cohabitación con alguna persona con sobrepeso y obesidad, del grupo de adolescentes con IMC normal, 21 de los 60 lo referían contra 37 del grupo con sobrepeso-obesidad, siendo esta diferencia estadísticamente significativa (p: 0,003); respecto al número de integrantes de la familia con sobrepeso-obesidad, de los adolescentes del grupo con peso normal, 16 manifestaron que eran de 1 a 2

Tabla 1. Datos generales por grupo

\begin{tabular}{|llrrr|}
\hline Edad & $\begin{array}{l}\text { Estado de } \\
\text { nutrición }\end{array}$ & Media & $\begin{array}{c}\text { Desviación } \\
\text { estándar }\end{array}$ & ** $^{*}$ \\
& $\begin{array}{l}\text { Normal } \\
\text { Obesidad }\end{array}$ & 16,8333 & 1,12245 & 0,224 \\
Peso & Normal & 55,9717 & 7,34226 & 0,012 \\
& Obesidad & 91,5350 & 10,32003 & \\
Talla & Normal & 1,6442 & 0,07146 & 0,018 \\
& Obesidad & 1,7337 & 0,09718 & \\
IMC & Normal & 20,6898 & 1,93068 & 0,013 \\
& Obesidad & 30,5390 & 2,87735 & \\
\hline
\end{tabular}

*p: U de Mann-Whitney. 
Tabla 2. Comparación de estructura, funcionalidad y etapa del ciclo familiar por grupos $\left(\chi^{2}\right)$

\begin{tabular}{|c|c|c|c|}
\hline & $\begin{array}{c}\text { Obesidad } \\
n=60(\%)\end{array}$ & $\begin{array}{c}\text { Control } \\
n=60(\%)\end{array}$ & Valor de p \\
\hline \multicolumn{4}{|l|}{ Composición (parentesco) } \\
\hline - Nuclear simple & $18(30)$ & $35(58,3)$ & 0,001 \\
\hline - Nuclear numerosa & $00(00)$ & $05 \quad(8,3)$ & \\
\hline - Monoparental & $01(1,6)$ & $06(10)$ & \\
\hline - Extensa & $28(46,6)$ & $07(11,6)$ & \\
\hline - Monoparental extendida & $00(00)$ & $06(10)$ & \\
\hline - No parental & $13(21,6)$ & $00(00)$ & \\
\hline - Monoparental extensa compuesta & $00(00)$ & $01 \quad(1,6)$ & \\
\hline \multicolumn{4}{|l|}{ Desarrollo } \\
\hline - Moderna & $30(50)$ & $34(56,6)$ & 0,464 \\
\hline - Tradicional & $30(50)$ & $26(43,3)$ & \\
\hline \multicolumn{4}{|l|}{ Integración } \\
\hline - Integrada & $21(35)$ & $28(46,6)$ & 0,421 \\
\hline - Semi-integrada & $21(35)$ & $18(30)$ & \\
\hline - Desintegrada & $18(30)$ & $14(23,3)$ & \\
\hline \multicolumn{4}{|l|}{ Demografía } \\
\hline - Rural & $22(36,6)$ & $18(30)$ & 0,234 \\
\hline - Urbana & $38(63,3)$ & $42(70)$ & \\
\hline \multicolumn{4}{|l|}{ Funcionalidad familiar } \\
\hline - Buena & $27(45)$ & $26(43,3)$ & 0,115 \\
\hline - Disfunción leve & $20(33,3)$ & $23(38,3)$ & \\
\hline - Disfunción moderada & $10(16,6)$ & $08(13,3)$ & \\
\hline • Disfunción severa & $03(05)$ & $03(05)$ & \\
\hline \multicolumn{4}{|l|}{ Etapa del ciclo familiar } \\
\hline - Extensión completa & $30(50)$ & $33(55)$ & 0,746 \\
\hline - Contracción & $26(43,3)$ & $22(36,6)$ & \\
\hline - Contracción completa & $04(6,6)$ & $05 \quad(8,3)$ & \\
\hline
\end{tabular}

integrantes y 5 de ellos que de 4 a 5 integrantes, contra 25 y 12 respectivamente del grupo de adolescentes con sobrepeso-obesidad, dichas diferencias no representaron significancia estadística (p: 0,560).

En el resto de las variables no se encontraron diferencias estadísticamente significativas en los grupos (Tabla 2).

\section{Discusión}

En este estudio se consideraron variables exclusivamente familiares, a las cuales no se les ha brindado el peso correspondiente debido a su dinámica; pero que contribuyen de gran manera en actitudes, estilos, gustos, costumbres y comportamientos de alimentación y actividad física que impactarán en el estado de nutrición y consecuentemente en la salud del adolescente.

El considerar sólo adolescentes de 15 a 18 años fue con la finalidad de controlar en lo posible la gran influencia hormonal, que aunque sus efectos continúan manifestándose es menor que a edad más temprana, además que los adolescentes ya presentan costumbres y gustos definidos en la alimentación y actividad física, los cuales persistirán hasta la edad adulta en $80 \%$ de ellos ${ }^{18}$. 
Además del período prenatal y el período de rebote de adiposidad (4 a 8 años de edad), la adolescencia se encuentra entre los tres estadios de crecimiento críticos para el desarrollo de obesidad persistente y de enfermedades asociadas en la edad adulta $^{19}$, el promedio de ganancia de peso durante la pubertad es de $14 \mathrm{~kg}$ en las hembras y de $15 \mathrm{~kg}$ en los varones, con una marcada diferencia en el aspecto físico ${ }^{20}$.

Respecto a la estructura familiar, con base en el parentesco, obtuvimos que la familia extensa (en la cual además de los padres cohabitan y conviven otros familiares con parentesco) fue la predominante en el grupo de adolescentes con sobrepeso-obesidad, y la nuclear simple en el grupo con peso normal; resulta importante mencionar que en la mayoría de los casos de familia extensa, el familiar conviviente fue la abuela, quien era la responsable de la preparación de los alimentos, debido a que ambos padres salían a trabajar; en el grupo con peso normal predominó la familia nuclear simple, y la madre era quien se encargaba de los aspectos de alimentación; la mayoría de las madres de este grupo no tenía un trabajo remunerado.

Resulta difícil comparar con otros estudios, debido a que son pocos los realizados en adolescentes y se consideran otras clasificaciones de estructura familiar; sin embargo, Kuntz y cols. en un estudio efectuado en Estados Unidos de Norteamérica reportaron que hasta $80 \%$ de los adolescentes con sobrepeso-obesidad eran originarios de familias monoparentales ${ }^{21,22}$.

En este tipo de investigaciones es importante considerar el contexto familiar normal según los usos y costumbres del área geográfica donde se vaya a realizar, así como utilizar escalas y clasificaciones validadas en poblaciones similares.

López y Macilla ${ }^{23}$ argumentan que la familia juega un papel determinante en la génesis de los problemas alimenticios y la obesidad puede ser proporcionada por una dinámica familiar disfuncional, en donde el individuo obeso funciona como un medio para aminorar los conflictos y tensiones que se propician por dicha dinámica; el sobrepeso y obesidad se correlacionan con una alta problemática familiar, dinámica semejante a la de las familias psicosomáticas.

Diferentes estudios reportan que los menores de edad con familias disfuncionales presentan mayor proporción de obesidad, explicando la alimentación como mecanismo de compensación de la ansiedad generada por los conflictos y tensiones de la dinámica. Es de relevancia mencionar que la funcionalidad familiar a través del APGAR se determina a través de la satisfacción del entrevistado con su vida familiar, siendo de utilidad para determinar si la familia es un recurso para el paciente o un factor que contribuye a la enfermedad, en este caso a la obesidad ${ }^{24}$. En este estudio no se encontraron diferencias estadísticamente significativas en la funcionalidad familiar.

Geográficamente se ha reportado una mayor prevalencia de obesidad en niños y adolescentes residentes del área urbana, debido a una mayor accesibilidad a comidas rápidas, ricas en carbohidratos, mayor exposición a la publicidad y poca vigilancia en la alimentación ${ }^{26}$, empero no encontramos diferencias significativas en este aspecto, es importante resaltar que en la región donde se desarrolló el estudio no existe un límite geográfico claro respecto a las zonas urbanas y rurales y la accesibilidad a alimentos, publicidad y demás factores es prácticamente igual, debido a las cortas distancias.

Los datos obtenidos coinciden con la mayoría de los estudios en que la presencia de un individuo en el grupo familiar con sobrepeso-obesidad, con o sin relación parental, representa un riesgo para desarrollar sobrepeso-obesidad en el niño $y$ el adolescente debido al reforzamiento de hábitos inadecuados de alimentación, patrones de repetición, disponibilidad de alimentación y percepción corporal ${ }^{25}$; en este estudio sólo la presencia resultó determinante; no así el número de individuos con obesidad en el grupo familiar, aunque el grupo de adolescentes con sobrepeso-obesidad convive con un mayor número de personas con sobrepeso-obesidad que el grupo con peso normal; la diferencia no resultó estadísticamente significativa.

La relevancia e impacto de este estudio radica en la consideración de aspectos contextuales del adolescente, los cuales están relacionados con su desarrollo desde el nacimiento y que sin duda impactarán en la vida adulta. Sin embargo, dado el dinamismo del contexto familiar y la influencia de factores externos para estudios futuros es importante considerar otras variables como la cultura, costumbres, nivel socioeconómico, estilo de vida y percepción de salud de todo el grupo familiar donde se desarrolla el adolescente. 


\section{Conclusión}

Existen diferencias en la estructura familiar de los adolescentes portadores de sobrepeso y obesidad en donde predomina la familia extensa en comparación con los adolescentes con peso normal quienes pertenecen a una familia nuclear.

\section{Referencias}

1. Domínguez-Vásquez P, Olivares S, Santos JL. Influencia familiar sobre la conducta alimentaria y su relación con la obesidad infantil. ALAN [revista en la Internet]. 2008 Sep [citado el 24 de mayo de 2012]; 58 (3): 249-255. Disponible en: http://www.scielo.org.ve/scielo.php?script=sci_arttext\&pid=S0004-06222008000300006\&l$\mathrm{ng}=\mathrm{es}$.

2. Espitia-Carrascal RE, Montes-Rotela M. Influencia de la familia en el proceso educativo de los menores del barrio de Costa Azul de Sincelejo (Colombia). Investigación y desarrollo 2009; 17 (1): 84-105.

3. Osorio-Rodríguez A, Álvarez-Mora A. Introducción a la salud familiar. En: Salas-Chávez A, Torres- Martínez R, Icaza Gurdian C, Carballo-Rosalbal M, editores. Curso especial de posgrado en gestión local en salud. San José, Costa Rica: 2004; p.11-69.

4. Busdiecker S, Castillo C, Salas I. Cambios en los hábitos de alimentación durante la infancia: una visión antropológica. Rev Chil Pediatr 2000; 71 (1): 5-11.

5. Suárez-Cuba MA. Temas de medicina familiar: El médico familiar y la atención a la familia. Rev Paceña Med Fam 2006; 3 (4): 95-100.

6. Huerta-González JL. PAC Medicina Familiar-1: Estudio de la salud familiar. Distrito Federal, México: Intersistemas; 1999.

7. Irigoyen Coria A. Nuevo Diagnóstico Familiar. 3Ed. Medicina Familiar Mexicana, México, 2005.

8. Bueno M, Sarría A. Exploración general de la nutrición. En: Galdó A, Cruz M, eds. Tratado de exploración clínica en pediatría. Barcelona: Masson, 1995: 587-600.

9. Narváez G, Narváez X. Índice de masa corporal (IMC). Nueva Visión y Perspectiva. Laboratorio de Evaluaciones Morfofuncionales LABEMORF. [en internet]. 2001 [consultado el 11 de marzo de 2012]. Disponible en: http://www.fac.org.ar/scvc/llave/PDF/narvaeze.pdf.

10. Silva H, Collipal E, Martínez C, Torres I. Analysis of BMI and Somatotype in a Sample of Adolescents were Overweight and Obesity in Temuco-Chile. Int. J. Morphol 2008; 26 (3): 707-11.

11. Zayas-Torriente GM, Chiong-Molina D, Díaz Y, Torriente-Fernández A, Herrera-Argüelles X. Obesidad en la infancia: Diagnóstico y tratamiento. Rev Cubana Pediatr 2002; 74 (3): 233-9.

12. Resultados de la Encuesta Nacional de Salud y Nutrición 2012 (ENSANUT 2012) [en internet] 2012 [consultado el 11 de abril de 2013] disponible en: http://ensanut. insp.mx/informes/ENSANUT2012ResultadosNacionales.pdf

13. Henry-Rodríguez M, Lazcano E, Hernández-Prado B, Oropeza Abúndez C. Instituto Nacional de Salud Pública. Encuesta Nacional de Salud y Nutrición 2006. Resultados por entidad federativa, Sonora. Cuernavaca, México: Instituto Nacional de Salud Pública-Secretaría de Salud, 2007.

14. Meléndez-Torres JM. La alimentación sonorense: factores que inciden en la alimentación de los niños. Centro de investigación en alimentación y desarrollo. [en internet]. 2010 [consultado el 20 de marzo de 2012]. Disponible en: http://www.ciadenlaciencia.com.mx/ vernoticias.php? artids $=627 \&$ categoria $1=28$

15. Magaña-Ruiz F, Ibarra-Ramírez J. Ruiz-García, Rodríguez-Orozco AR. Hay relación entre estado nutricional estimado por antropometría y tipología familiar, en niños mexicanos entre 1 y 4 años. Nutr Hosp 2009; 24 (6): 751-62.

16. Ortega-Vélez MI. Nutrición: La dieta Sonorense. Instituto de educación Sonora-Arizona. [en internet]. 2012 [consultado el 20 de marzo de 2012]. Disponible en: http://www.iesa.gob.mx/sonarida/22/dieta-sonorense. htm

17. Sandoval-Godoy SA, Domínguez-Ibáñez S, Cabrera-Murrieta A. De golosos y tragones están llenos los panteones: cultura y riesgo alimentario en Sonora. Estud. Soc. [en internet] 2009. [consultado el 5 de mayo de 2012]. Disponible en: http:// www.scielo.org.mx/scielo.php?script=sci_arttext\&pi$\mathrm{d}=$ S0188-45572009000300007\&lng=es\&nrm=iso >.

18. Guo SS, Roche AF, Chumlea WC, Gardner JC, Siervogel RM. The predictive value of childhood body mass index values of overweight at age 35 years. Am J Clin Nutr 1994; 59: 810.

19. Freedman D, Ketel L, Dietz W, Srinivasan S. Relationship of childhood obesity to coronary heart disease risk factors: The Bogalusa Heart Study. Pediatrics 2001; 108 (3): 712-8.

20. Dasha Nicholls. ABC of adolescence Eating disorders and weight problems. Clinical review BMJ 2005; 330: 950-3.

21. Kuntz B, Lampert T. Socioeconomic factors and obesity. Dtsch Arztebl Int 2010; 107 (30): 517-22.

22. Huffman FG, Kanikireddy S, Patel M. Parenthood-A contributing factor to childhood obesity. Int. J. Environ. 
Res. Public Health 2010; 7 (7): 2800-10.

23. López AX, Mancilla DI. La estructura familiar y la comunicación en obesos y normopeso. Revista Mexicana de Psicología. 2000: 17 (1): 6575.

24. Huerta González JL. Medicina Familiar, La familia como unidad de estudio, 1 Ed. Biblioteca Alfil, 2005.

25. Mullis R, Blair S, Aronne L, Bier D, Denke MA, Donato $\mathrm{K}$, et al. Obesity, a Worldwide Epidemic Related to Heart Disease and Stroke: Group IV: Prevention/Treatment. Circulation 2004; 110: e484-8. 